Original Research

\title{
Controllable Cyclopalladated Polythiophene Imine Monolayer by Self- Assembly, Hybrid Doping and Electrochemical Polymerization: A Simple Way to Enhance Activity and Stability
}

\author{
Louguangshu Huang ${ }^{1,+}$, Hui Liu ${ }^{2,+}$, Xiaoxia Xue ${ }^{1}$, Wuduo Zhao ${ }^{1}$, Tiesheng $\mathrm{Li}^{1,{ }^{*}}$
}

1. College of Chemistry, Zhengzhou University, Zhengzhou 450001, Henan Province, P.R. China; EMails: $\quad$ 623250349@qq.com; $18337166254 @ 163 . c o m ; \quad$ zhaowuduo@163.com; Its34@zzu.edu.cn

2. School of Chemistry and Chemical Engineering, Zhengzhou Normal University, Zhengzhou 450044, Henan Province, P.R. China; E-Mail: Ih610@163.com

+ These authors contributed equally to this work.

* Correspondence: Tiesheng Li; E-Mail: Its34@zzu.edu.cn

Academic Editor: Jacques Muzart

Special Issue: Advanced in Palladium-Catalyzed Reactions

Catalysis Research

2022, volume 2 , issue 1

doi:10.21926/cr.2201003
Received: December 13, 2021

Accepted: January 23, 2022

Published: February 03, 2022

\begin{abstract}
In this article, an approach to generate self-assembly cyclopalladated polythiophene imine monolayers (denoted as ITO@Pd-CPTIMs) is described. The monolayers were fabricated by combining self-assembly (SA), hybrid doping (HD), and electrochemical polymerization (ECP) called SA-HD-ECP. The catalytic activity and stability of the polymerized monolayers in the Suzuki coupling reaction were improved by modulating the structure and morphology in hybrid doping using different thiophene derivatives, concentrations, and scanning numbers during electrochemical polymerization. Morphological changes in the catalytic surface associated with catalytic activity were investigated. ITO@Pd-PTF could improve catalytic activity with a higher TON value $\left(45000 \mathrm{~mol} / \mathrm{mol}_{\text {cat }}\right)$ and attain recycling ability at least 10 times.
\end{abstract}

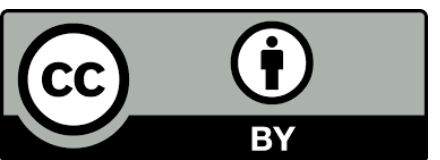

(C) 2022 by the author. This is an open access article distributed under the conditions of the Creative Commons by Attribution License, which permits unrestricted use, distribution, and reproduction in any medium or format, provided the original work is correctly cited. 


\section{Keywords}

Cyclopalladated thiophene imine; coupling reaction; self-assembly; hybrid doping; electrochemical polymerization

\section{Introduction}

Thiophene chemistry has been a well-established field of research, and polythiophenes are considered a representative class due to their interest in $\pi$-conjugation, which are used for incorporating the metallic units resulting in different behaviors $[1,2]$. The palladium complex is considered as one of the most utilized and versatile compounds [3], and Schiff-based Pd complexes are usually employed for building new structures in catalytic applications [4-8]. Thiophene derivatives and their complex films can be fabricated using the self-assembly or Langmuir-Blodgett (LB) method, which has attracted great attention because it can be used to obtain the surfaces tailored with specific chemical and physical properties $[9,10]$. In our previous studies, ordered cyclopalladated ferrocenylimine (CPFI) LB films were fabricated [11, 12], which exhibited better catalytic activities [13-16]. In addition, polymerized ordered thiophene LB films were prepared by combining electrochemical polymerization (ECP) with LB films [17]. Variations in surface morphologies of thiophene derivatives due to electropolymerization were considered very important factors [18-22]. However, the control of fabrication on self-assembly cyclopalladated thiophene imine monolayers covalently bonded on supports by combining self-assembly, hybrid doping, and electrochemical polymerization (ECP) was still a challenge, which is considered important for the future development of catalyst materials.

In this paper, polymerized cyclopalladated polythiophene imine monolayers linked with supports were fabricated using SA-HD-ECP, and their catalytic performances in coupling reactions were investigated. The objective of this study was to generate a self-assembly polymeric monolayer with catalytic sites attached to support surfaces by the electrochemical method, which was an attempt in the preparation of self-assembly catalytic monolayers.

\section{Materials and Methods}

\subsection{Materials and Measurements}

\subsubsection{Chemicals}

Chemicals were obtained from commercial sources. The solvents were distilled using appropriate drying agents under nitrogen. All silicon wafers and indium tin oxide (ITO) conductive glasses $(<10 \Omega / \mathrm{sq})$ were purchased from commercial sources.

\subsubsection{Measurement}

Water contact angles (WCA) were measured on a commercial HARKE-SPCAX1 instrument. X-ray photoelectron A SPM-9500 J3 (Shimadzu Corporation, Japan) was employed for AFM measurements in the air at ambient temperatures. The Pd content in the catalysts before and 
after the cross-coupling reactions was measured by inductively coupled plasma atomic emission spectroscopy (ICP-AES) with an ICAP 6000 Series (Thermo Scientific). Cyclic Voltammetry (CV) curves were obtained using a $\mathrm{CH}$ 1650A; the experiments were performed in three-electrode cells in $0.5 \mathrm{M} \mathrm{HCl}$. Platinum was used as a counter electrode, and $\mathrm{Ag} / \mathrm{AgCl} /(0.1 \mathrm{M}) \mathrm{KCl}$ was used as a reference electrode. By using a Thermo Scientific DXR Raman microscope with an excitation laser wavelength of $532 \mathrm{~nm}$, Raman spectra were recorded. An ESCALab220i-XL electron spectrometer(VG Scientific) with $300 \mathrm{~W}$ Al Ka radiations was used to carry out the X-ray photoelectron spectroscopy (XPS) analysis.

\subsection{General Operation}

\subsubsection{Hydrophilic Treatment}

The silicon or ITO wafers were added to the mixed solvent of concentrated nitric acid and concentrated sulfuric acid (v:v =2:1) and heated for 1-1.5 $\mathrm{h}$ until they were boiled. Then, the wafers were washed with deionized water, acetone, and chloroform. The ITO glasses were ultrasonically washed with acetone, ammonia, ethanol, and pure water sequentially. The ITO glasses were used for electrochemical study, WCA, AFM, XPS measurement, and catalysis.

\subsubsection{Fabrication of Self-Assembly Cyclopalladated Imine Thiophene Monolayer}

The imine thiophene monolayer polymerized electrochemically under different experimental conditions (ITO) was mixed with a solution of $\mathrm{Li}_{2} \mathrm{PdCl}_{4}$ for a certain time period and then taken out and washed with methanol several times.

\subsubsection{Inductively Coupled Plasma-Atomic Emission Spectrometry (ICP-AES) Analysis}

After the completion of the reaction, the substrate linked with the catalyst was removed, and the reaction mixture was centrifuged and broken down with nitrolysis, where the $\mathrm{Pd}$ concentration was measured by using ICP-AES.

\subsubsection{Recovery and Reuse of the Modified Substrates in the Suzuki Coupling Reaction}

Optimal reaction conditions were employed for each recycling run. The substrates were then removed from the reactor, washed with methanol, dried under vacuum, and reused without further purifications.

\subsubsection{Suzuki Cross-Coupling Reactions}

In a small round-bottom flask, haloarenes $(0.25 \mathrm{mmol})$, arylboronic acid $(0.3 \mathrm{mmol})$, catalyst $\left(2.5 \mathrm{~cm} \times 1.0 \mathrm{~cm} \times\left(\mathrm{Pd}\right.\right.$ content $\left.\left./ \mathrm{cm}^{2}\right)\right)$, and TBAB $(0.3 \mathrm{mmol})$ were mixed with base $(0.3 \mathrm{mmol})$ and ethanol/deionized water $(4: 1,5 \mathrm{~mL})$. First, the reaction mixture was stirred, and then it was extracted with ethyl acetate three times. In order to obtain the target molecule, the combined filtrate was concentrated, and the residue was chromatographed with a column of silica gel by using an eluent. 


\section{Results and Discussion}

\subsection{Preparation and Characterization of ITO@Pd-CPTIMs}

According to the requirements for palladacycle formation, thiophene derivatives containing Schiff-base group having the ability to coordinate more transition metals were designed.

As shown in Figure 1, polymerized cyclopalladated 3-thiopheneimine derivative monolayers (ITO@Pd-CPTIMs) were fabricated by using SA-HD-ECP [4, 17]. The fabrication process of ITO@Pd-CPTIMs was characterized by using WCA, AFM, CV, and XPS [23-25], and the catalytic monolayer with various surfaces was controlled by tuning the doping substrate or applying different potentials.
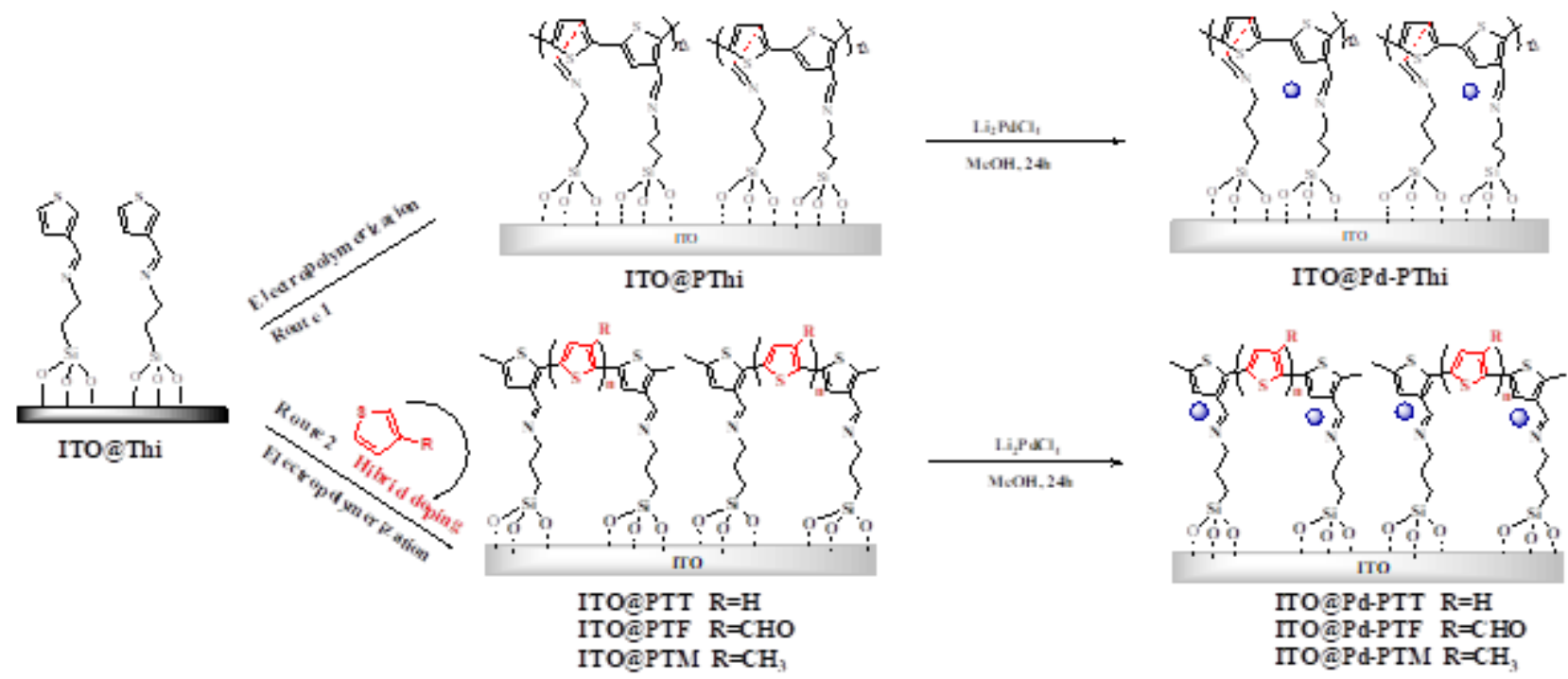

Figure 1 Preparation routes of ITO@Pd-CPTIMs with doping thiophene derivatives (route 2, ITO@PTT was prepared by doping thiophene coordinated with palladium in order to form ITO@Pd-PTT; ITO@PTF was prepared by doping 3thiophenecarboxaldehyde(3-formylthiophene) coordinated with palladium in order to form ITO@Pd-PTF, and ITO@PTM was prepared by doping 3-methylthiophene coordinated with palladium in order to form ITO@Pd-PTM) or without doping (route 1, ITO@PThi was prepared by electrochemical polymerization without doping and coordinated with palladium to yield ITO@Pd-PThi).

WCA changes in different preparation steps of ITO@Pd-PTIMs supported on ITO were measured (Figure 2) so that the wettability of different functionalized surfaces could be elucidated. Due to the formation of ITO@Pd-PThi, the WCA value of ITO@PThi varied from $64^{\circ}$ to $54^{\circ}$ after being treated with $\mathrm{Li}_{2} \mathrm{PdCl}_{4}$ (Scheme 1, route 1). In the case of route 2 , due to the formation of ITO@Pd-PTF, ITO@Pd-PTT, and ITO@Pd-PTM, respectively, the WCA values of ITO@PTF, ITO@PTT, and ITO@PTM varied from $71^{\circ}$ to $55^{\circ}$, from $72^{\circ}$ to $57^{\circ}$, and from $65^{\circ}$ to $55^{\circ}$ after being treated with $\mathrm{Li}_{2} \mathrm{PdCl}_{4}$, which indicated that the surface configuration significantly changed due to ECP. 


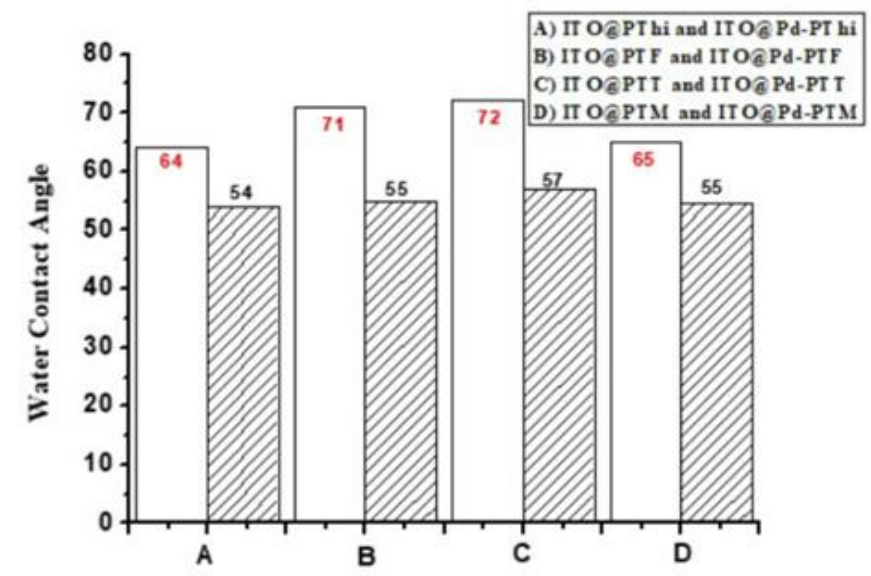

Figure 2 Water contact angles of (A) ITO@PThi and ITO@Pd-PThi, (B) ITO@PTF and ITO@Pd-PTF, (C) ITO@PTT and ITO@Pd-PTT, and (D) ITO@PTT and ITO@Pd-PTT.

In order to compare with ITO@Pd-Thi monolayer, AFM images in the fabrication process of ITO@Pd-Thi were recorded (Figure S1), and the roughness values are given in Table S1.

AFM images of the prepared ITO@Pd-PThi, ITO@Pd-PTT, ITO@Pd-PTF, and ITO@Pd-PTM are shown in Figure $3 a-d$, respectively. The image shows an irregular packing structure associated with different roughness values, which were $47.5 \mathrm{~nm}, 26.5 \mathrm{~nm}, 24.9 \mathrm{~nm}$, and $26.3 \mathrm{~nm}$, respectively (Table 1). Among them, a larger roughness of ITO@Pd-PThi with island-like features was observed, which might be caused by the change in the orientation and arrangement of molecules in the monolayer during electrochemical polymerization. As shown in Figure 3c, the roughness of ITO@Pd-PTF was found to be smaller than that of ITO@Pd-PTT and ITO@Pd-PTE. For the isolated nucleation and island, the average roughness was measured as $24.9 \mathrm{~nm}$ (Figure 3c), indicating that a more uniform image was formed [17].

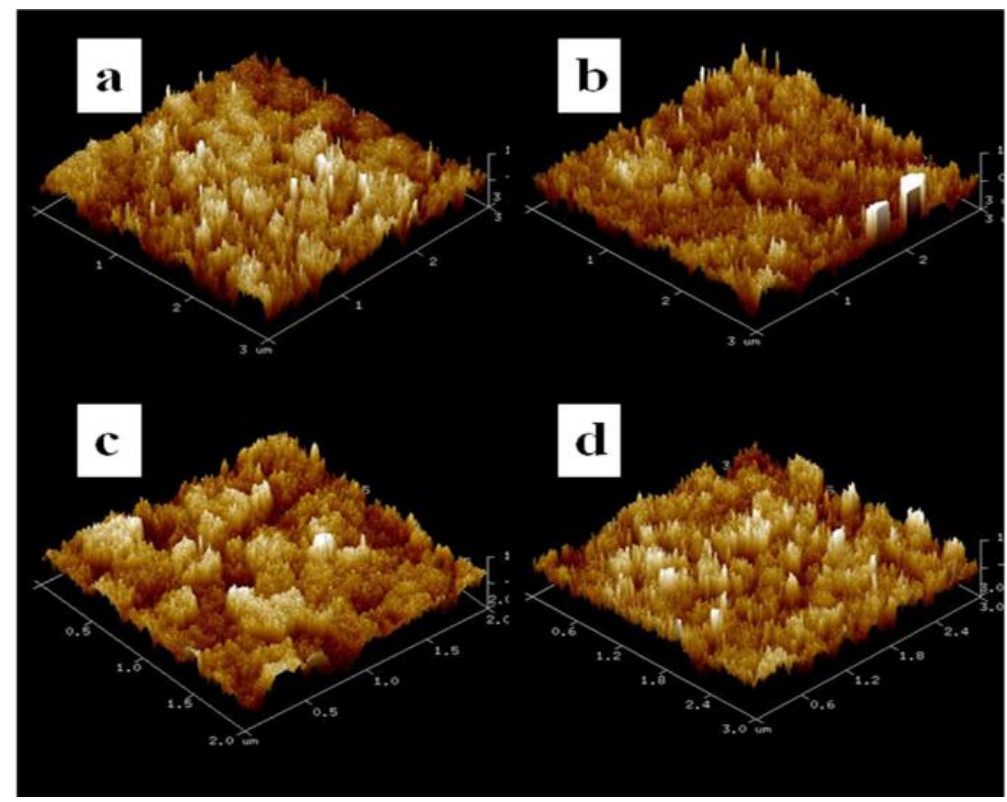

Figure 3 AFM images of surface topographies of the prepared palladium catalytic monolayers linked with ITO: (a) ITO@Pd-PThi, (b) ITO@Pd-PTT, (c) ITO@Pd-PTF, and (d) ITO@Pd-PTM (ITO@Pd-Thi is shown in Figure S1d). 
Table 1 Summary of the RMS of the prepared Pd catalysts on ITO from Figure 2.

\begin{tabular}{llllll}
\hline Catalyst & ITO@Pd-Thi & ITO@Pd-PThi & ITO@Pd-PTT & ITO@Pd-PTF & ITO@Pd-PTM \\
\hline RMS & 29.4 & 47.5 & 26.5 & 24.9 & 26.3 \\
(nm) & & & & \\
\hline
\end{tabular}

Electrochemical characterizations of ITO@Thi monolayer with different hybrid doping were carried out by cyclic voltammetry (CV) to detect fabrication of ITO@PThi, ITO@PTT, ITO@PTF, and ITO@PTM monolayer (Figure 4). As shown in Figure 4A for ITO@PThi, the first scan showed the electroactivity at +0.6 to $+0.4 \mathrm{~V}$, whereas the next scan showed a little increase in the current response, indicating no better tendency to electropolymerization for ITO@Thi. As shown in Figure 4B for ITO@PTT, the first scan showed monomer oxidation at $+0.10 \mathrm{~V}$ and a reductive procedure at $0.20 \mathrm{~V}$. In the second scan, new oxidation was observed at $+0.16 \mathrm{~V}$, indicating the oxidation of polymerized film. ITO@PTT showed a similar tendency and similar shapes compared to that of ITO@PThi. However, the potential values were higher than that of ITO@PThi, indicating that ITO@PTT with doping thiophene showed a similar tendency but was easier to electropolymerize than that of ITO@PThi.
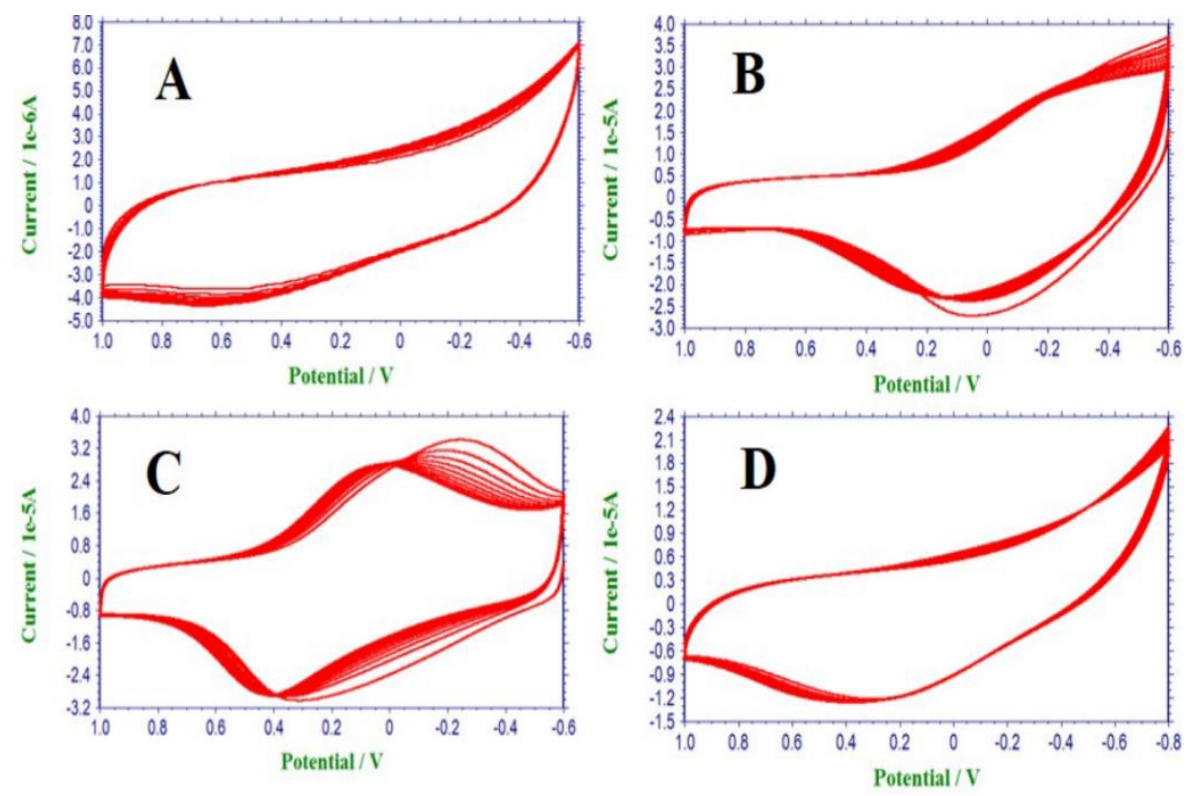

Figure 4 Repeated potential scan electropolymerization of ITO@Thi: (A) at $0.1 \mathrm{M}$ $\mathrm{mol} \cdot \mathrm{L}^{-1} \mathrm{CH}_{2} \mathrm{Cl}_{2}$-TBAP, containing (B) $0.1 \mathrm{M}$ thiophene, (C) $0.1 \mathrm{M}$ 3-thiophene formaldehyde, and (D) $0.1 \mathrm{M}$ 3-methyl thiophene in $0.1 \mathrm{M} \mathrm{mol} \cdot \mathrm{L}^{1} \mathrm{CH}_{2} \mathrm{Cl}_{2}$-TBAP with a scan rate of $100 \mathrm{mV} \cdot \mathrm{s}^{1}$ (10 cycles were typically used).

In the first scan, when doped with 3-thiophenecarboxaldehyde (Figure 4C), ITO@PTF showed a sharper oxidation peak at $+0.38 \mathrm{~V}$, and a reductive peak at $-0.06 \mathrm{~V}$. A new oxidation peak appeared at $+0.35 \mathrm{~V}$ due to the oxidation of the polymerized monolayer in the second scan. It was clearly evident that an increase in the current response of oxidation and reversible redox was observed. The increased intensity of these redox peaks for electroactivity and the polymerized monolayer with increasing scans was found consistent with that the polymerized film with a larger 
surface area than that of ITO@Thi. A similar result for ITO@PTM was also obtained, as shown in Figure 4D, which showed no better tendency to be electropolymerized. The results of electropolymerization suggested that polymerization of these self-assembly monolayers with different thiophene backbones on ITO could be performed under experimental conditions. In addition to the monomer on the ITO surface, the extended thienylene linkages were also observed in monolayers. It was likely that the resulting ITO@PTT, ITO@PTF, and ITO@PTM films propagated from the two adjacent imine thienyl units coupled with doped thiophene derivatives, which was proved by the redox waves, increased in currents during the successive cycle due to the growth of the polymerized monolayer [18]. It was due to the fact that thio-units in films acted as proper sites with electrochemical polymerization; thus, it resulted in a lower electrical potential [25]. This method could form different arrays and structures in the polymerized monolayer, and thus better catalytic monolayers could be identified.

As shown in Figure S2, the Raman spectra of ITO@Thi, ITO@PThi, ITO@PTT, ITO@PTF, and ITO@PTM (Figure S2(A)) and ITO@Pd-PThi, ITO@Pd-PTT, ITO@Pd-PTF, and ITO@Pd-PTM (Figure S2(B)) were measured. The stretching vibration and the absorption at $2989-2864 \mathrm{~cm}^{-1}$ were attributed to $-\mathrm{CH}_{2}-$. Peaks at $1603 \mathrm{~cm}^{1}, 1447 \mathrm{~cm}^{1}$, and $1338 \mathrm{~cm}^{1}$ were designated for the imine group, $C_{\alpha}=C_{\beta}$, and $C_{\beta}-C_{\beta}$ of thiophene for ITO@Thi before polymerization (Figure S2 $\left.(A)-a\right)[26,27]$. The characteristic peak of the imine group showed no change, and other two peaks of ITO@PThi, ITO@PTT, ITO@PTF, and ITO@PTM at $1447 \mathrm{~cm}^{1}$ and $1338 \mathrm{~cm}^{1}$ shifted after electrochemical polymerization, indicating that the polymerization occurred (Figure $2 S(A)-b-e)$. The characteristic peak of the imine group shifted to $1595 \mathrm{~cm}^{1}$ and the other two peaks also shifted due to the palladium coordination with imine groups when the polymerized monolayer coordinated with $\mathrm{Li}_{2} \mathrm{PdCl}_{4}$ (Figure S2(B)-a-d).

The XPS spectra of ITO@Pd-PThi, ITO@Pd-PTT, ITO@Pd-PTF, and ITO@Pd-PTM were measured (Figure S2), presenting the peaks attributed to $\mathrm{Si}, \mathrm{C}, \mathrm{N}, \mathrm{O}, \mathrm{S}, \mathrm{Pd}$, and $\mathrm{Cl}$. The highresolution XPS spectra of ITO@Pd-PThi, ITO@Pd-PTT, ITO@Pd-PTF, and ITO@Pd-PTM were measured (Figure 5), and all showed $\mathrm{Pd}(3 \mathrm{~d} 5 / 2)$ and $\mathrm{Pd}(3 \mathrm{~d} 3 / 2)$ peaks at $343.2 \mathrm{eV}$ and $338.0 \mathrm{eV}$ assigned to $\mathrm{Pd}^{2+}$, indicating the presence of $\mathrm{Pd}^{2+}$ in these films (Figure 5A-D). The positions of peaks with their monolayers are listed in Table S2. As summarized in Table S3, the Pd contents of the fabricated ITO@Pd-PThi, ITO@Pd-PTT, ITO@Pd-PTF, and ITO@Pd-PTM films were measured by using the ICP-AES analysis. The characteristic results of WCA, AFM, CV, and XPS proved that ITO@Pd-CPTIMs were fabricated. 

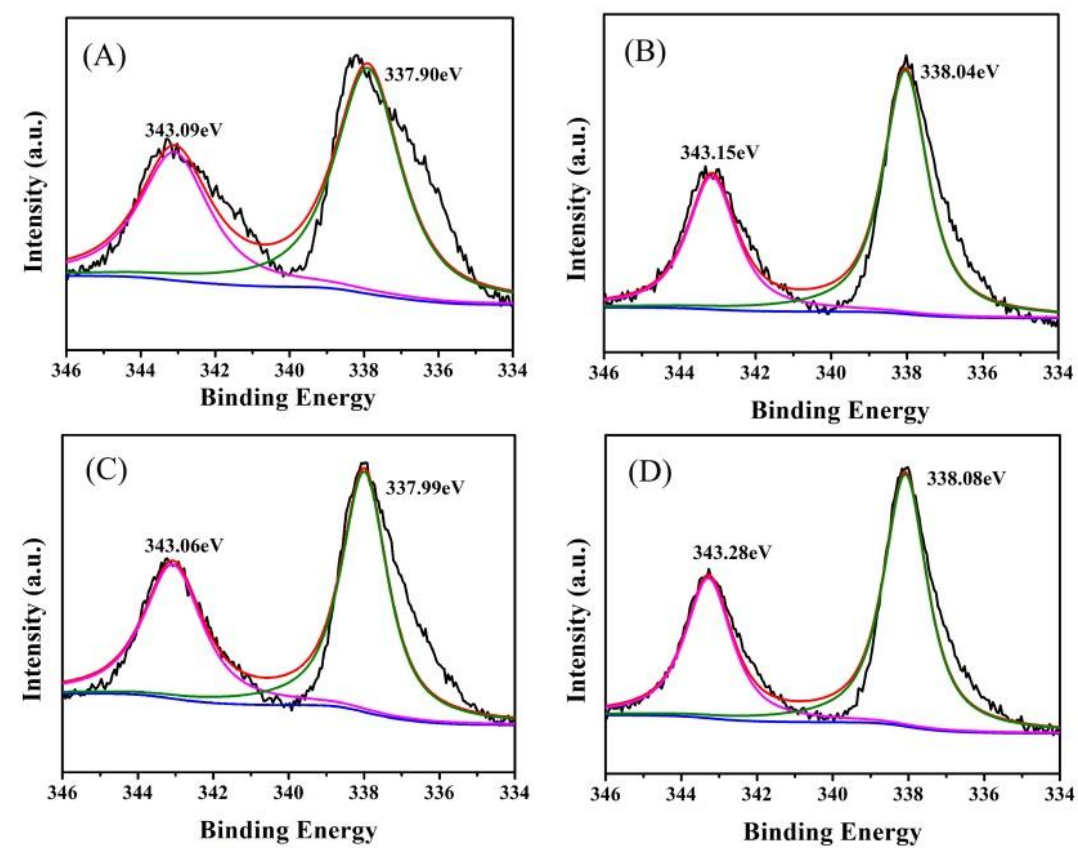

Figure 5 High-resolution XPS spectra of $\mathrm{Pd}(3 \mathrm{~d} 5 / 2)$ and $\mathrm{Pd}(3 \mathrm{~d} 3 / 2)$ peaks in $(A)$ ITO@Pd-PThi, (B) ITO@Pd-PTT, (C) ITO@Pd-PTF, and (D) ITO@Pd-PTM films.

\subsection{Catalytic Activity in the Suzuki Coupling Reaction}

In order to catalyze the Suzuki coupling reaction with phenylboronic acid and $p$-bromotoluene using the screened $\mathrm{K}_{3} \mathrm{PO}_{4} / \mathrm{EtOH}: \mathrm{H}_{2} \mathrm{O}(4: 1) / 60{ }^{\circ} \mathrm{C}$ [27], the electropolymerized monolayers were selected. ITO@Pd-PTT and ITO@Pd-PTF exhibited higher yields than those of ITO@Pd-PThi and ITO@Pd-PTM (Figure 6). It was evident that the morphology and array of catalytic surface modulated by doping thiophene derivatives (having electron withdrawing group, electron donating group and steric effect) affected their catalytic activity.

To further examine the relationship between the activity and electropolymerization by CV, ITO@Pd-PTT and ITO@Pd-PTF were selected to investigate the effect on catalytic activity in different polymeric conditions such as scan runs and doping concentrations of thiophene derivatives. 


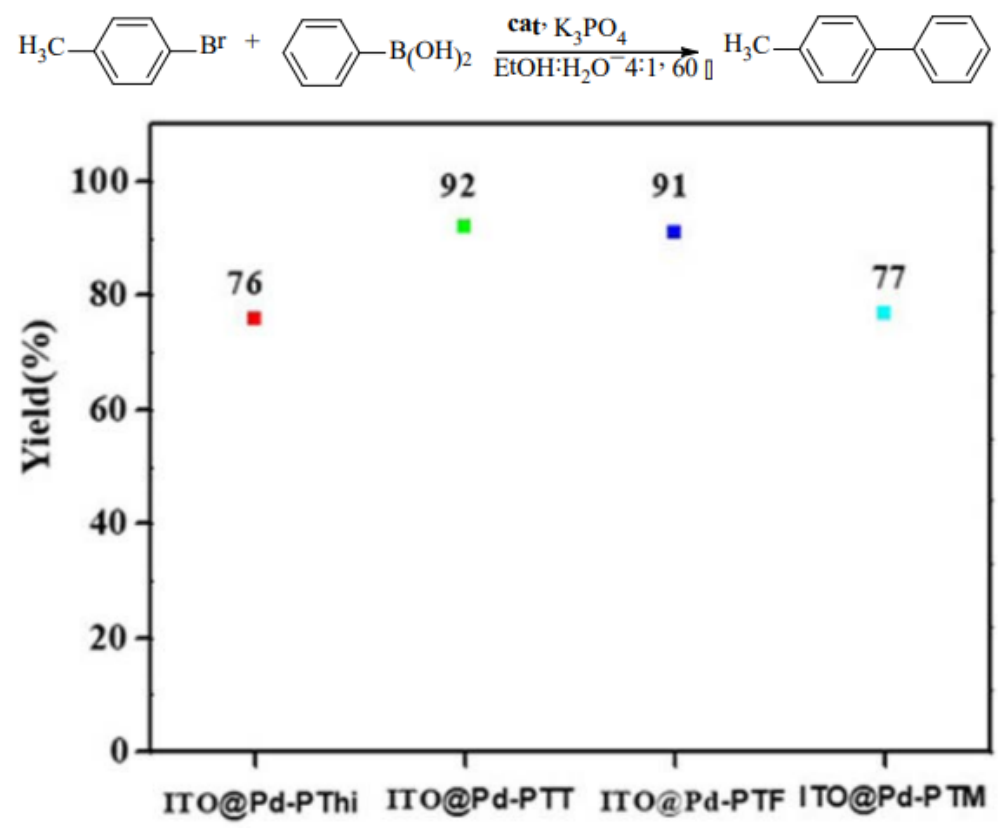

Figure 6 Yields of the Suzuki coupling reaction catalyzed by ITO@Pd-PThi, ITO@Pd-PTT, ITO@Pd-PTF, and ITO@Pd-PTM. (The polymerization was performed by adding the same concentration (10 $\mathrm{mM})$ to 3-thiophenecarboxaldehyde, thiophene, and 3methyl-thiophene (10 scan runs).)

When 10 mM of 3-thiophenecarboxaldehyde was added for ITO@Pd-PTF (Figure 7 (red column)), the yield reached up to $91 \%$. A yield of $99 \%$ could be obtained by doping $20 \mathrm{mM}$ of 3-thiophenecarboxaldehyde. However, the yields decreased when the concentration reached more than $20 \mathrm{mM}$, which was the reason that the catalytic surface would be covered by too much polymer produced. In order to investigate the effect of scans on catalytic activity under certain concentrations of doped thiophene derivatives, in which the highest yield was obtained with 10 runs, ITO@Pd-PTF was also fabricated with different scans $(5,10,20$, and 30 scans), where the highest yield was obtained with 10 runs. For ITO@Pd-PTT (Figure 7, black column), the addition of $10 \mathrm{mM}$ of thiophene and 10 cycle scans were considered as the optimized conditions. 


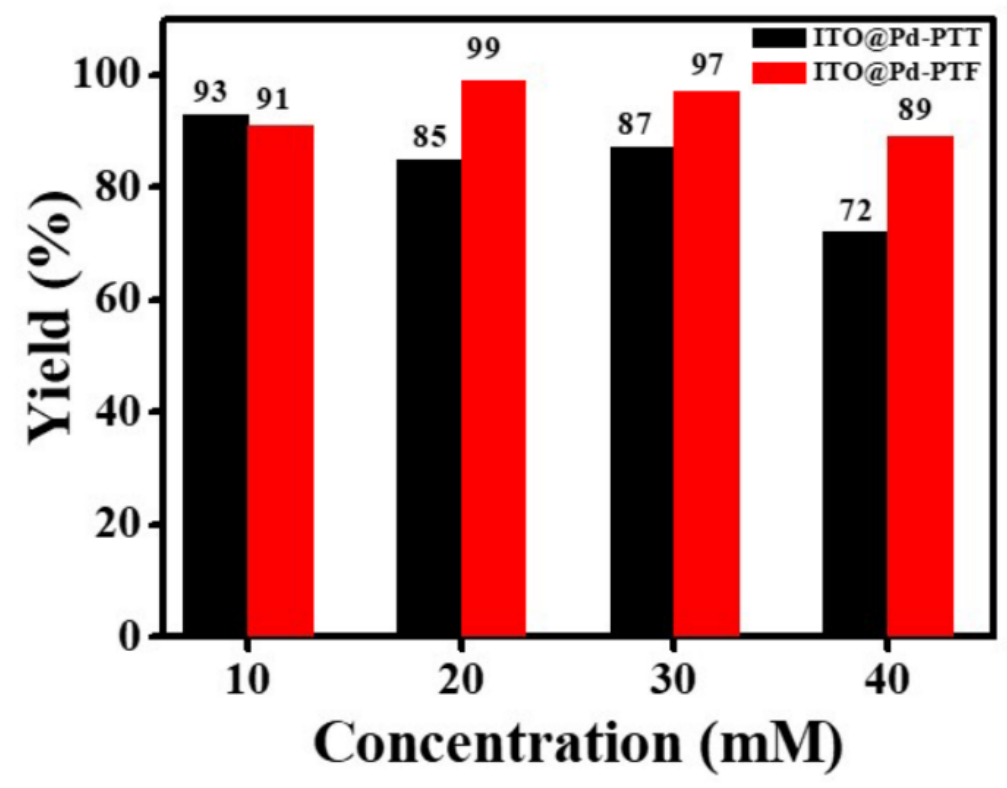

Figure 7 Yields of ITO@Pd-PTF (red bar) and ITO@Pd-PTT (black bar) with different concentrations of 3-thiophenecarboxaldehyde and thiophene at the polymerization process in the catalysis of the Suzuki coupling reaction (10 scan runs).

As compared to experiments presented in Table 2, certain polymerization conditions were applied, and the activity could be enhanced, such as ITO@Pd-PTF. Some of them showed loss of activity because the fabricated arrays were not found suitable for catalyzing as compared to ITO@Pd-Thi, such as ITO@Pd-PThi and ITO@Pd-PTM, indicating that the proper dopant was considered an important factor due to the steric effect during polymerization.

Table 2. The Suzuki coupling reaction of phenylboronic acid with 4-bromotoluene catalyzed by catalysts (blank ITO was used in the solution ${ }^{\mathrm{a}}$ ).

\begin{tabular}{llll}
\hline Catalyst & $\begin{array}{l}\text { Pd content } \\
\left(10^{9} \mathrm{~mol} \cdot \mathrm{cm}^{2}\right)\end{array}$ & Yield $(\%)^{\mathrm{b}}$ & TON \\
\hline ITO@Pd-PThi & 1.08 & 76 & 35185 \\
ITO@Pd-PTF & 1.10 & 99 & 45000 \\
ITO@Pd-PTT & 1.11 & 93 & 41891 \\
ITO@Pd-PTM & 1.05 & 77 & 36666 \\
ITO@Pd-Thi & 1.12 & 90 & 40178 \\
ITO & - & - & - \\
\hline
\end{tabular}

a-Bromotoluene $(0.25 \mathrm{mmol}), \mathrm{PhB}(\mathrm{OH})_{2}(0.30 \mathrm{mmol}), \mathrm{K}_{3} \mathrm{PO}_{4}(0.30 \mathrm{mmol})$, and $\mathrm{EtOH} / \mathrm{H}_{2} \mathrm{O}(4: 1$, $5.0 \mathrm{~mL})$; temperature: $60^{\circ} \mathrm{C}, 24 \mathrm{~h}$; Cat. $(2.5 \mathrm{~cm} \times 1 \mathrm{~cm} \times 1 \mathrm{~cm})$.

${ }^{\mathrm{b}}$ Isolated yield.

The screening of substrates was also investigated in detail by using ITO@Pd-PTF under optimized conditions (see Table S4). The results revealed that ITO@Pd-PTF showed an efficient activity in the Suzuki coupling reaction, where bromobenzene with the electron-donating group or 
the electron-withdrawing group could present medium or higher yields (entries 1-10). However,the target molecule could not be obtained in the case of chlorobenzene (entries 11-13) because the oxidative addition of chloroarenes to palladium (0) was too slow to develop the catalytic cycle due to the higher band energy of the $\mathrm{C}-\mathrm{Cl}$ bond. No products or lower yields were observed in the reaction of 4-bromotoluene with arylboronic acid derivatives (entries 14-18). The fact that whether can the trans-metalation of arylboronic acid with oxidative addition intermediate under basic conditions proceed is related to the electronic structure of arylboronic acid. Arylboronic acids with the electron-withdrawing groups often resulted in medium or lower yields due to the easy cleavage of the C-B bond with the base (entry 18). In the case of the electron-donating group, different yields were obtained according to the electronic and stericeffects that play a key role in the metal-translation step.

As shown in Figure 8, certain structure and morphology that could afford efficient active sites were considered key factors, whereas the mechanism of this polymerization was unclear. Comparisons of the reported similar studies are presented in Table S5, indicating that a higher TON value of this work could be obtained.

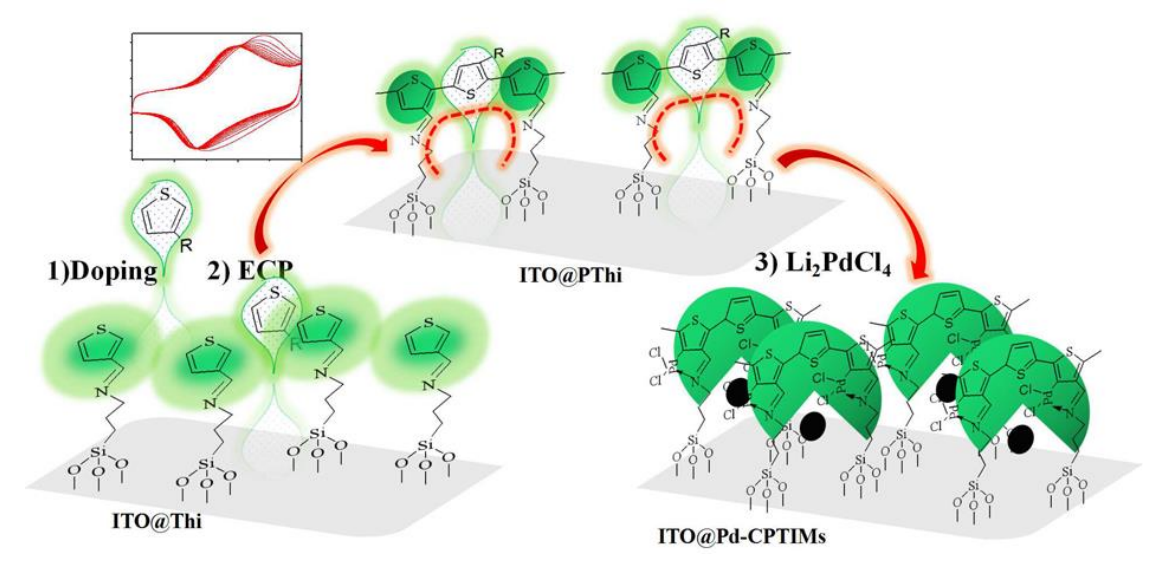

Figure 8 Different arrays and structures in electrochemical-polymerized monolayers.

Recycling ability is considered an important factorbecause the recycling and stability of catalysts play an important role in industrial applications [13, 28-31]. As shown in Figure 7, recycling of these catalytic monolayers is also investigated. The recycling ability of the ITO@Pd-Thi catalyst exhibited lower stability because the yield of subsequent coupling reactions dropped to $52 \%$ in the fourth run (Figure 9, blue bar). ITO@Pd-PTF exhibited the highest stability as compared to ITO@Pd-PTT and ITO@Pd-Thi due to the electropolymerization process. ITO@Pd-PTF and ITO@Pd-PTT could be reused the seventh and sixth times, respectively (Figure 9, red, black bar). It was observed that ITO@Pd-PTF and ITO@Pd-PTT could improve the catalytic properties under optimized electropolymeric conditions by doping the thiophene derivatives. However, sharp decreases in the yield were observed after the eighth and seventh runs due to the deactivation during the reaction process [32, 33]. Enhanced stability was most likely attributed to better mechanical properties and morphologies due to electrochemical polymerization [34-36]. 


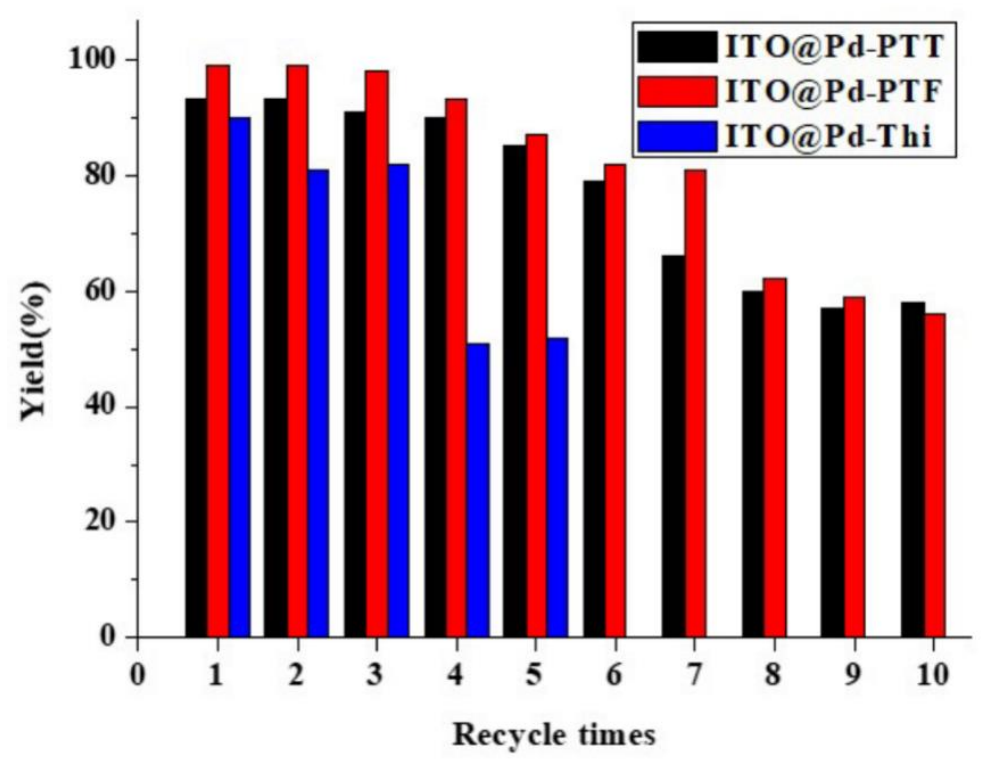

Figure 9 Reusability of ITO@Pd-Thi, ITO@Pd-PTT, and ITO@Pd-PTF.

\section{Conclusions}

An approach denoted as SA-HD-ECP was developed to fabricate and screen diverse catalytic monolayers on the surfaces of ITO. Polymerization at different ratios of thiophene derivatives with self-assembly monolayer containing thiophene group resulted in different surfaces with modulated catalytic performances. Self-assembly, doping, and ECP might solve the problem of poor activity and stability of some catalytic monolayers with proper doping under electropolymerization conditions. The generation of modified array on surface of catalyst should be made straight forward by copolymerizing thiophene or other compounds, which might be suitable for enhancing activity and stability.

\section{Acknowledgments}

The authors gratefully acknowledge the Henan Natural Science Foundation of China (192102210046) for their financial support. Thank Prof. Luyuan Mao, Zhengzhou University for AFM measurement.

\section{Author Contributions}

Prof.Tiesheng Li designed and analysis; Dr Wuduo Zhao measurement; MS Louguangshu Lou, Hui Liu and Xiaoxia Xue synthesis and analysis.

\section{Competing Interests}

The authors have declared that no competing interests exist.

\section{Additional Materials}

The following additional materials are uploaded at the page of this paper. 
1. Figure S1: AFM images of surface topographies of prepared palladium catalyst supported on ITO and its precursors. a) ITO@OH (hydrophilic treatment), b) ITO@APTES (silanization), c) ITO@Thi (thienyl Schiff base graft) and d) ITO@Pd-Thi (Pd-thienyl Schiff based complex grafted).

2. Figure S2: Raman spectra of (A) a, ITO@Thi; b, ITO@PThi; c, ITO@PTT; d, ITO@PTF; e, ITO@PTM. (B)a, ITO@Pd-PThi; b, ITO@Pd-PTT; c, ITO@Pd-PTF; d, ITO@Pd-PTE.

3. Figure S3: XPS survey spectrum of (A) ITO@Pd-PThi, (B) ITO@Pd-PTT, (C) ITO@Pd-PTF and (D) ITO@Pd-PTM.

4. Table S1: Data of Rms of ITO@OH, ITO@APTES, ITO@Thi and ITO@Pd-Thi monolayers.

5. Table S2: Positions of BE peaks in catalyst monolayers.

6. Table S3: Summary of Pd contents of catalysts prepared.

7. Table S4: Suzuki coupling reaction of aryl halide with phenylboronic acid.

8. Table S5: Comparisons of the results in Suzuki reaction catalyzed by the catalysts supported on different supports.

\section{References}

1. Mishra A, Ma CQ, Bauerle P. Functional oligothiophenes: Molecular design for multidimensional nanoarchitectures and their applications. Chem Rev. 2009; 109: 1141-1276.

2. Cinar ME, Ozturk T. Thienothiophenes, dithienothiophenes, and thienoacenes: Syntheses, oligomers, polymers, and properties. Chem Rev. 2015; 115: 3036-3140.

3. Suzuki A. Carbon-carbon bonding made easy. Chem Commun. 2005: 4759-4763. doi: 10.1039/B507375H.

4. Bhunia S, Koner S. Heterogeneous stille and sonogashira coupling reaction over palladium anchored mesoporous silica catalyst. Indian J Chem. 2011; 50A: 1380-1387.

5. Huang PP, Xue ZQ, Li TS, Liu ZY, Wei DH, Liu MH, et al. Investigation on electron distribution and synergetic to enhance catalytic activity in bimetallic Ni (II)/Pd (II) molecular monolayer. ChemCatChem. 2018; 10: 5141-5153.

6. Liu H, Xue XX, Li TS, Wang J, Xu WJ, Liu MH, et al. A simple, recyclable, and self-assembled palladium (II)-alkyl schiff base complex for Suzuki coupling reaction: Chain length dependence and heterogeneous catalysis. RSC Adv. 2016; 6: 84815-84824.

7. Wang LH, Huang PP, Yang J, Li TS, Mao LY, Liu MH, et al. Fabrication and catalytic properties of ordered cyclopalladated diimine monolayer: Investigation on catalytic mechanism. RSC Adv. 2018; 8: 31860-31867.

8. Albano VG, Bandini M, Moorlag C, Piccinelli F, Pietrangelo A, Tommasi S, et al. Electropolymerized pd-containing thiophene polymer: A reusable supported catalyst for cross-coupling reactions. Organometallics. 2007; 26: 4373-4375.

9. Bandini $M$, Pietrangelo A, Sinisi R, Umani-Ronchi A, Wolf MO. New electrochemically generated polymeric pd complexes as heterogeneous catalysts for Suzuki cross-coupling reactions. Eur J Org Chem. 2009; 2009: 3554-3561.

10. Zhang L, Colella NS, Liu F, Trahan S, Baral JK, Winter HH, et al. Synthesis, electronic structure, molecular packing/morphology evolution, and carrier mobilities of pure oligo/poly(alkylthiophenes). J Am Chem Soc. 2013; 135: 844-854.

11. Fu ZH, Li TS, Mu B, Mao LY, Li GQ, Xu WJ, et al. Cyclopalladated ferrocenylimine self-assembly films for Suzuki coupling reaction. J Mol Catal A: Chem. 2012; 363-364: 200-207. 
12. Mu B, Li TS, Li CH, Liu PP, Shang W, Wu YJ. Langmuir-blodgett films of cyclopalladated ferrocenylimine: Preparation, characterization, and application in Suzuki coupling reaction. Tetrahedron. 2009; 65: 2599-2604.

13. Zhao N, Li TS, Zhai Z, Qiu JJ, Xu WJ, Liu H, et al. Cyclopalladated arylimine self-assembly films for Suzuki reaction. ChemCatChem. 2013; 5: 1481-1489.

14. Fu Z, Zhang N, Liu J, Li T, Xu W, Wang F, et al. N-hydroxymethyl acrylamide polymer brush and its application in catalyzing coupling reaction. J Colloid Interf Sci. 2013; 394: 409-418.

15. Fu ZH, Liu J, He XH, Li TS, Wu YJ. The recyclable cyclopalladated ferrocenylimine self-assembly catalytic film and investigation of its role in the mechanism of heterogeneous catalysis. RSC Adv. 2014; 4: 26413-26420.

16. Zhao N, Wang F, Zhou ML, Li TS, Liu H, Xu WJ, et al. Preparation, characterization and catalytic activity of amphiphilic cyclopalladated aryl imines and their langmuir-blodgett films. Chinese J Catal. 2013; 34: 1583-1588.

17. Zou KK, Liu H, Li TS, Chen PL, Liu MH, Wu YJ. Electrochemical polymerized and assemblied cyclopalladated bithiophene imine for catalyzing coupling reaction: A modern strategy to enhance catalytic activity. RSC Adv. 2015; 5: 16654-16663.

18. Reddinger JL, Reynolds JR. Site specific electropolymerization to form transition-metalcontaining, electroactive polythiophenes. Chem Mater. 1998; 10: 1236-1243.

19. Koh WC, Chandra P, Kim DM, Shim YB. Electropolymerized self-assembled layer on gold nanoparticles: Detection of inducible nitric oxide synthase in neuronal cell culture. Anal Chem. 2011; 83: 6177-6183.

20. So RC, Carreon-Asok AC. Molecular design, synthetic strategies, and applications of cationic polythiophenes. Chem Rev. 2019; 119: 11442-11509.

21. Bai $S, H u$, Zeng $Q$, Wang $M$, Wang LS. Variations in surface morphologies, properties, and electrochemical responses to nitro-analyte by controlled electropolymerization of thiophene derivatives. ACS Appl Mater Interfaces. 2018; 10: 11319-11327.

22. Wang M, Das MR, Li MS, Boukherroub R, Szunerits S. "Clicking" thiophene on diamond interfaces. preparation of a conducting polythiophene/diamond hybrid material. J Phys Chem C. $2009 ; 113: 17082-17086$.

23. Siu T, Yekta S, Yudin AK. New approach to rapid generation and screening of diverse catalytic materials on electrode surfaces. J Am Chem Soc. 2000; 122: 11787-11790.

24. Aaronson BD, Garoz-Ruiz J, Byers JC, Colina A, Unwin PR. Unwin, electrodeposition and screening of photoelectrochemical activity in conjugated polymers using scanning electrochemical cell microscopy. Langmuir. 2015; 31: 12814-12822.

25. Rider DA, Harris KD, Wang D, Bruce J, Fleischauer MD, Tucker RT, et al. Thienylsilane-modified indium tin oxide as an anodic interface in polymer/fullerene solar cells. ACS Appl Mater Interfaces. 2009; 1: 279-288.

26. Liu H, Li TS, Xue XX, Xu WJ, Wu YJ. The mechanism of a self-assembled Pd(ferrocenylimine)-Si compound-catalysed Suzuki coupling reaction. Cata Sci Technol. 2016; 6: 1667-1676.

27. Li L, Xue XX, Sun YM, Zhao WD, Li TS, Liu MH, et al. Self-assembly palladacycle thiophene imine monolayer-Investigating on catalytic activity and mechanism for coupling reaction. Chem Res Chin Univ. 2020; 36: 821-828. 
28. Shang WL, Zeng XF, Li TS, Xu WJ, Wei DH, Liu MH, et al. Controlled distribution of active centre to enhance catalytic activity of ordered Pd/Co catalytic nano-monolayer. J Catal. 2019; 376: 228-237.

29. Ren RR, Huang PP, Zhao WD, Li TS, Liu MH, Wu YJ. A new ternary organometallic Pd (II)/Fe (III)/Ru (III) self-assembly monolayer: The essential ensemble synergistic for improving catalytic activity. RSC Adv. 2021; 11: 1250-1260.

30. An QQ, Wang LH, Bi S, Zhao WD, Wei DH, Li TS, et al. Sandwich structured aryl-diimine Pd (II)/Co (II) monolayer-Fabrication, catalytic performance, synergistic effect and mechanism investigation. Mol Catal. 2021; 501: 111359.

31. Molnár Á, Papp A. Catalyst recycling-A survey of recent progress and current status. Coord Chem Rev. 2017; 349: 1-65.

32. Xue ZQ, Huang PP, Li TS, Qin PX, Xiao D, Liu MH, et al. A novel "tunnel-like" cyclopalladated arylimine catalyst immobilized on graphene oxide nano-sheet. Nanoscale. 2017; 9: 781-791.

33. Huang PP, Song ER, Sun YM, Li TS, Wei DH, Liu MH, et al. Schiff-based Pd (II)/Fe (III) bimetallic self-assembly monolayer-preparation, structure, catalytic dynamic and synergistic. Mol Catal. 2019; 469: 75-86.

34. Xu LY, Wei DL, Chen YX, Li RB. A feasible electrochemical method for tuning coatings wettability from super hydrophilicity to super hydrophobicity. Appl Surf Sci. 2016; 385: 80-87.

35. Xie CG, Li HF, Li SQ, Wu J, Zhang ZP. Surface molecular self-assembly for organophosphate pesticide imprinting in electropolymerized poly ( $p$-aminothiophenol) membranes on a gold nanoparticle modified glassy carbon electrode. Anal Chem. 2010; 82: 241-249.

36. Alex PC, Gunther B, Ullrich S. Thiophene-based microporous polymer networks via chemical or electrochemical oxidative coupling. Macromolecules. 2015; 48: 6816-6824.

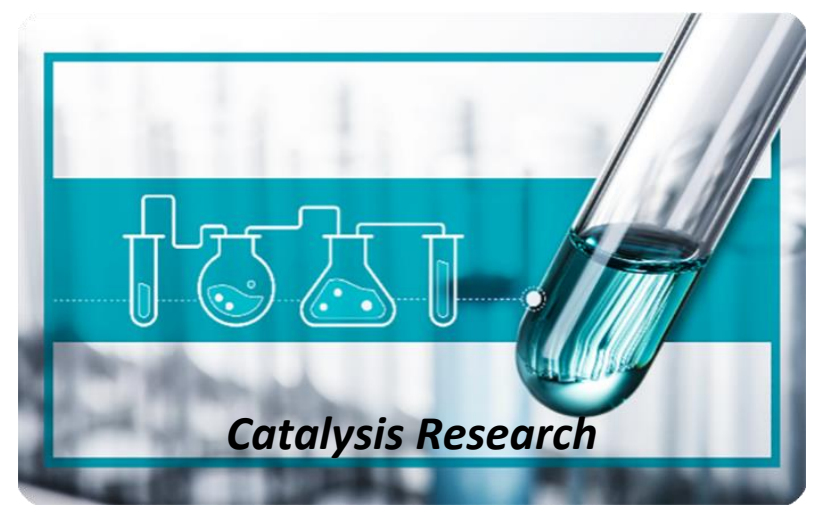

Enjoy Catalysis Research by:

1. Submitting a manuscript

2. Joining in volunteer reviewer bank

3. Joining Editorial Board

4. Guest editing a special issue

For more details, please visit: http://www.lidsen.com/journals/cr 